\title{
Results of surveillance for infections with Shiga toxin- producing Escherichia coli (STEC) of serotype O104:H4 after the large outbreak in Germany, July to December 2011
}

C Frank (frankc@rki.de) ${ }^{1}$, A Milde-Busch1, D Werber ${ }^{1}$

1. Department for Infectious Disease Epidemiology, Robert Koch Institute, Berlin, Germany

Citation style for this article:

Frank C, Milde-Busch A, Werber D. Results of surveillance for infections with Shiga toxin-producing Escherichia coli (STEC) of serotype $\mathrm{O}_{104}$ : $\mathrm{H}_{4}$ after the large outbreak in Germany, July to December 2011. Euro Surveill. 2014;19(14):pii=20760. Available online: http://www.eurosurveillance.org/ViewArticle. aspx?Articleld $=20760$

After the massive outbreak of infections with Shiga toxin-producing Escherichia coli (STEC) of serotype 0104: $\mathrm{H}_{4}$ in Germany in the summer of 2011, post-outbreak surveillance for further infections with this type of STEC was maintained until the end of 2011. This surveillance was based on national mandatory reporting of STEC infections and the associated complication of haemolytic uraemic syndrome (HUS), as well as on data obtained from a questionnaire. Between the outbreak's end (5 July) and 31 December 2011, a total of 33 post-outbreak cases were recorded. Post-outbreak cases occurred with diminishing frequency towards the year's end and resembled the outbreak cases in many respects, however the proportion of HUS among all post-outbreak cases was smaller than during the outbreak. Two thirds of the post-outbreak cases were likely infected by contact with known outbreak cases. Both laboratory and nosocomial spread was noted in this period. No post-outbreak case recalled sprout consumption as a potential source of infection. The scarcity of information conveyed by the nonculture tests routinely used in Germany to diagnose STEC made linkage of post-outbreak cases to the outbreak difficult. Though post-outbreak surveillance demonstrated the outbreak strain's potential for lengthy chains of transmission aided by prolonged shedding, our results and continued routine surveillance until the end of 2013 do not support the notion, that the outbreak strain has been able to establish itself in the German environment.

\section{Introduction}

A large outbreak of gastroenteritis caused by Shiga toxin-producing Escherichia coli (STEC) occurred in Germany in 2011 [1-3]. It was caused by a STEC of the rare serotype $0104: \mathrm{H}_{4}$, positive for a Shiga toxin 2 gene $\left(s t x_{2}\right)$, negative for $s t x_{1}$ and intimin (eae), and a carrier of the extended-spectrum beta-lactamase (ESBL) antibiotic resistance trait [4-6]. More than 20\% of the recognised outbreak cases developed the life threatening post-diarrhoeal sequela of haemolytic uraemic syndrome (HUS). Considering the large number of ensuing outbreak cases of HUS, this was the largest known STEC-associated outbreak worldwide. Uncharacteristically for STEC infections, mostly adults were affected by all disease types (i.e. both gastroenteritis only and that complicated by HUS). Consumption of fenugreek sprouted seeds was identified as the most likely source of infection for primary outbreak cases [1]. Particularly later in the outbreak, person-to-person transmission and food-borne outbreaks associated with infected food handlers [7] also took place. Cases also occurred in other countries than Germany, but the majority of such cases, as well as most German cases, were associated with residence or temporary stay in the north of Germany; in France a satellite outbreak occurred in June 2011, also attributed to consumption of fenugreek sprouts [8]. The outbreak peaked on 22 May 2011. After a lapse of three weeks without newly diagnosed cases, the outbreak was declared over after 4 July 2011.

STEC infection is reportable in Germany and STEC is diagnosed predominantly by nonculture tests. In addition, clinical diagnosis of 'enteropathic', i.e. post-diarrhoeal HUS is separately reportable. The Robert Koch Institute (RKI), Germany's national level infectious disease surveillance hub, coordinated an intensified postoutbreak surveillance for additional 'post-outbreak' cases of the infection arising from 5 July through 31 December 2011. The primary goal of this surveillance was to verify the absence of post-outbreak cases of the infection associated with sprout consumption and to receive early warning of a potential resurgence of the outbreak. Also the study aimed to assess, whether the outbreak strain had managed to establish itself in the German environment, continuing to cause new infections. Results of this post-outbreak surveillance are presented here. We analysed post-outbreak cases ascertained in the German surveillance system for 
Overview of the post-outbreak cases ${ }^{a}$ by case definition category, sex, age and period of disease onset, Germany, 5 July-31 December $2011(n=33)$

\begin{tabular}{|c|c|c|c|c|c|c|c|c|}
\hline \multirow{2}{*}{ Case definition (CD) category } & \multirow{2}{*}{ HUS } & \multirow{2}{*}{$\begin{array}{c}\text { STEC- } \\
\text { gastroenteritis }\end{array}$} & \multirow{2}{*}{ Asymptomatic } & \multirow{2}{*}{ Female } & \multirow{2}{*}{ Adults $^{b}$} & \multicolumn{3}{|c|}{ Disease onset in calendar weeks } \\
\hline & & & & & & $27-35$ & $36-44$ & $45-52$ \\
\hline Confirmed $(n=22)$ & 0 & 17 & 5 & 15 & 19 & 15 & 2 & 0 \\
\hline Probable $(n=7)$ & 1 & 2 & 4 & 3 & 4 & 3 & 0 & 0 \\
\hline Possible $(n=4)$ & 4 & $N A^{c}$ & $N A^{c}$ & 2 & All per CD & 1 & 3 & 0 \\
\hline Total $(n=33)$ & 5 & 19 & 9 & 20 & 23 & 19 & 5 & o \\
\hline
\end{tabular}

NA: not applicable; HUS: haemolytic uraemic syndrome; STEC: Shiga toxin-producing Escherichia coli.

Weeks 27-35 correspond to 5 Jul-4 Sep; weeks 36-44 correspond to 5 Sep-6 Nov; weeks 45-52 correspond to 7 Nov-31 Dec.

a Cases in question were detected by intensified post-outbreak surveillance in the aftermath of the large outbreak of gastroenteritis caused by STEC that occurred in Germany in $2011[1-3]$.

b $\geq 18$ years of age.

Not applicable because according to the possible case definition only cases of HUS among adults ( $\geq 18$ years of age) are counted.

infectious diseases by clinical presentation, period of disease onset and their likely context of exposure.

\section{Methods}

Analysis rested on the regular legally-prescribed notification of laboratory-confirmed (at least finding of Stx or stx-gene in the laboratory, serotyping information is optional) cases of STEC-gastroenteritis, or cases of gastroenteritis with an epidemiological (exposure) connection to a laboratory-confirmed case of STEC gastroenteritis. In addition, cases of enteropathic HUS are notified separately as clinical entities, optionally supported by laboratory confirmation of STEC. Cases of HUS are only counted as such (not as STEC gastroenteritis). Cases notified to local health departments are then passed on electronically via the federal states to the national level database hosted by the RKI [9]. In addition, during the months following the outbreak until year's end, a small paper questionnaire was filled in addition to the case notification, based on local health departments' interviews with the cases, and sent on to the RKI. The questionnaire focused on food exposures (analysed here was sprout consumption only) and contacts to previously infected persons. Occupational exposures (in a laboratory or a healthcare environment) were related to the RKI spontaneously, whenever health departments considered this the most likely context of exposure.

As completeness of laboratory diagnosis with respect to the outbreak strain varied, cases were grouped by the quality of microbiological evidence of infection with the outbreak strain (serotype 0104: $\mathrm{H}_{4}$, st $x_{2}$ positive, st $x_{1}$ negative, eae negative, ESBL positive) into 'confirmed', 'probable' and 'possible'. Timewise, as the outbreak was declared over as of 5 July, cases considered to be post-outbreak cases were those with disease onset from 5 July through 31 December 2011, or - if a date of onset was unknown or the case asymptomatic
- those notified to the local authorities in calendar weeks 29 through 52 of 2011 (data as of 16 July 2012).

- Confirmed post-outbreak cases had infections with STEC 0104 (serogroup reported) for which microbiological details (regarding stx-type, eae, and ESBL status), if available, were compatible with the outbreak strain, irrespective of clinical presentation (HUS, gastroenteritis without HUS or asymptomatic).

- Probable post-outbreak cases had STEC-infections without serogroup information, but for which microbiological details (regarding stx-type, eae, and ESBL status), if available, were compatible with the outbreak strain, irrespective of clinical presentation (HUS, gastroenteritis without HUS or asymptomatic), only if they were contact persons to known outbreak cases or confirmed post-outbreak cases.

- Possible post-outbreak cases were other cases of post-diarrhoeal HUS among adults (age $\geq 18$ years) without known serogroup, but with microbiological details (regarding stx-type, eae, and ESBL status), if available, compatible with an infection with the outbreak strain.

We excluded infections with STEC 0104 where available microbiological details (st $x_{1}$ positivity, eae positivity, ESBL negativity) argued against infection with the outbreak strain, but briefly describe them below.

\section{Results}

We ascertained 33 post-outbreak cases according to the criteria outlined above. Confirmed were 22, consisting of 17 post-outbreak cases of gastroenteritis, but none of HUS, and five asymptomatic post-outbreak cases of STEC 0104 infection. In addition, there were three symptomatic (2 cases of gastroenteritis only, 1 with HUS) and four asymptomatic probable post-outbreak 


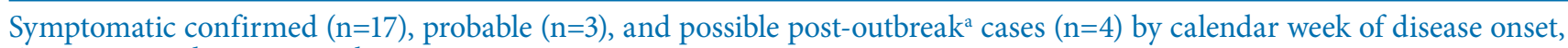
Germany, 5 July-31 December 2011

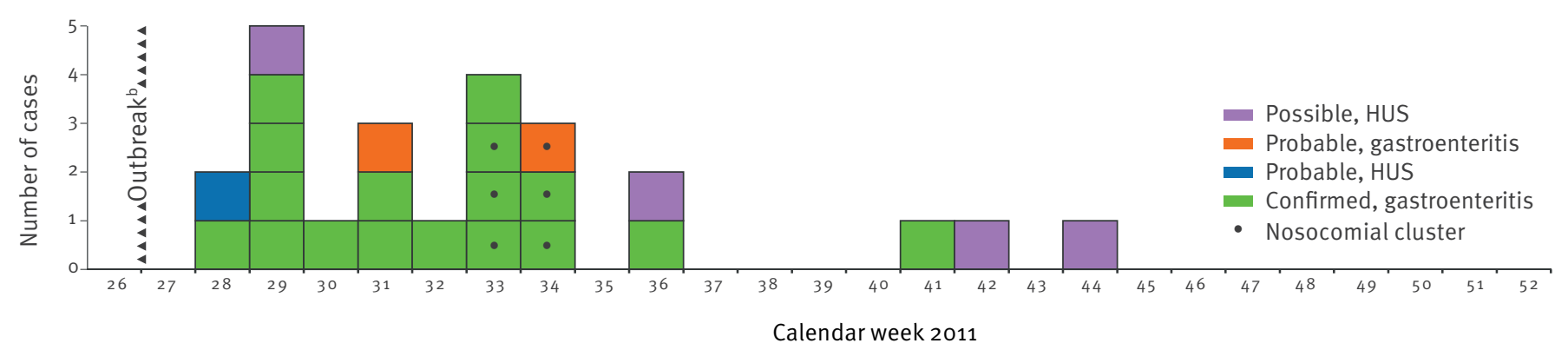

HUS: haemolytic uraemic syndrome; STEC: Shiga toxin-producing Escherichia coli.

a The cases depicted were detected by intensified post-outbreak surveillance in the aftermath of the large outbreak of gastroenteritis caused by STEC that occurred in Germany in $2011[1-3]$.

b The vertical line made up by the triangles indicates the declared end of the outbreak.

cases. Four HUS cases among adults were counted as possible post-outbreak cases (Table 1).

Three persons infected with a STEC 0104 strain were identified and not included in the study because microbiological findings were not compatible with infection with the outbreak strain: Two infections were with ESBL-negative strains of STEC $0_{104}$, and were in part already briefly mentioned elsewhere [10]. These were detected in adult residents of Germany after their return from travel to Turkey. Another case of gastroenteritis was caused by a st $x_{1}$-only positive STEC 0104 strain in a small child.

The majority of the 33 post-outbreak cases (Table 1, Figure 1) were female $(n=20)$ and adults $(n=26) ; 19$ of the 24 symptomatic post-outbreak cases had disease onset from 5 July through 4 September, and five from 5 September through 6 November - none occurred thereafter. In terms of geographical distribution, 27 of the 33 post-outbreak cases resided in the six German states most affected by the outbreak (Bremen, Hamburg, Lower Saxony, Mecklenburg-Western Pomerania, North Rhine-Westphalia, Schleswig-Holstein). Two thirds of the infections were likely caused by direct or indirect (laboratory workers) contact with recognised outbreak cases (Table 2). For the remainder (one third) the context of exposure remained unclear. Among them, seven arose from the most affected states. None of the postoutbreak cases recalled sprout consumption.

Included among the 33 post-outbreak cases were four with secondary ( 2 staff, 1 fellow patient, 1 relative, who washed the index patient's soiled clothes at home) and two with tertiary infections (household contacts of the aforementioned secondary cases) in a nosocomial cluster associated with a colonoscopy performed on an elderly woman. All had gastroenteritis, but not HUS.
The primary patient was an outbreak gastroenteritis case (disease onset in early June), who apparently was still shedding STEC 0104: $\mathrm{H}_{4}$ at the time of the procedure. Also included are three independently arising post-outbreak cases of gastroenteritis caused by STEC $\mathrm{O}_{104}$ in laboratory workers handling stools presumptively containing STEC 0104 .

\section{Discussion}

The 2011 STEC 0104:H4 outbreak was the most severe documented foodborne outbreak in Germany. It was accompanied by heightened anxiety and put a tremendous strain on the medical care system, especially nephrological treatment capacities $[11,12]$ and on public health resources. In consequence, after the declared end of the outbreak the RKI continued enhanced surveillance in order to quickly discover new flare-ups of the infection.

During the surveillance period, 22 confirmed post-outbreak cases occurred where infection with the outbreak strain was either confirmed or at least likely. In addition seven probable and four possible post-outbreak cases were notified in Germany. Most of these post-outbreak cases were household contacts to outbreak cases but for some the transmission route remained unclear. None of the post-outbreak cases remembered sprout consumption. Frequency of new post-outbreak cases was highest in the two months after the outbreak and then petered out in the autumn, with the last confirmed post-outbreak case patient's disease onset in the first half of October (week 41). The post-outbreak cases identified largely matched outbreak cases in terms of the predominance of adults and the female sex, and in terms of main occurrence in the area most affected by the outbreak [2]. This outcome may at least partially reflect the surveillance, which was likely more intense in the outbreak area and more frequently resulted in 


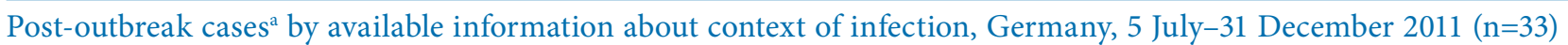

\begin{tabular}{|c|c|c|c|c|c|c|c|c|}
\hline \multirow[b]{2}{*}{$\begin{array}{l}\text { Case } \\
\text { definition } \\
\text { category }\end{array}$} & \multirow[b]{2}{*}{ Disease type } & \multicolumn{3}{|c|}{ Likely context of exposure } & \multicolumn{2}{|c|}{ Context of exposure unknown } & \multirow{2}{*}{$\begin{array}{l}\text { Sprout } \\
\text { consumption } \\
\text { recalled } \\
\text { by post- } \\
\text { outbreak } \\
\text { cases }\end{array}$} & \multirow[b]{2}{*}{ Total } \\
\hline & & Laboratory & $\begin{array}{l}\text { Hospital, care } \\
\text { home }\end{array}$ & $\begin{array}{l}\text { Private context, } \\
\text { household }\end{array}$ & $\begin{array}{l}\text { Residence in the } \\
\text { German federal } \\
\text { states most } \\
\text { affected by the } \\
\text { outbreak }^{\mathrm{b}}\end{array}$ & $\begin{array}{l}\text { Residence } \\
\text { elsewhere } \\
\text { in } \\
\text { Germanyc }\end{array}$ & & \\
\hline \multirow[t]{2}{*}{ Confirmed } & Gastroenteritis & 3 & $\begin{array}{c}4 \\
\text { (incl. } 3 \text { secondary } \\
\text { NC cases) }\end{array}$ & $\begin{array}{c}4 \\
\text { (incl. } 1 \text { secondary } \\
\text { and } 1 \text { tertiary NC } \\
\text { cases) }\end{array}$ & 5 & 1 & 0 & 17 \\
\hline & Asymptomatic & 0 & 1 & 3 & o & 1 & o & 5 \\
\hline \multirow{3}{*}{ Probable } & HUS & o & 0 & 1 & $N A^{d}$ & $N A^{d}$ & o & 1 \\
\hline & Gastroenteritis & o & 0 & $\begin{array}{l}2 \text { (incl. } 1 \text { tertiary } \\
\text { NC case) }\end{array}$ & $N A^{d}$ & $N A^{d}$ & 0 & 2 \\
\hline & Asymptomatic & 0 & 0 & 4 & $N A^{d}$ & $N A^{d}$ & 0 & 4 \\
\hline Possible & $\begin{array}{c}\text { HUS only (per } \\
\text { case definition) }\end{array}$ & o & 0 & o & 2 & 2 & 0 & 4 \\
\hline Total & All types n (\%) & $3(9)$ & $5(15)$ & $14(42)$ & $7(21)$ & $4(12)$ & o (o) & $33(100)$ \\
\hline
\end{tabular}

Incl.: includes; HUS: haemolytic uraemic syndrome; NA: not applicable; NC: cases constituting one nosocomial cluster; STEC: Shiga toxin-producing Escherichia coli.

a Cases in question were detected by intensified post-outbreak surveillance in the aftermath of the large outbreak of gastroenteritis caused by STEC that occurred in Germany in 2011 [1-3].

b Most-affected states: Bremen, Hamburg, Lower Saxony, Mecklenburg-Western Pomerania, North Rhine-Westphalia, Schleswig-Holstein. Includes two cases who likely acquired infection during travel outside of Germany.

d According to the case definition, probable cases had to have known contact to outbreak cases.

extended microbiological examination of outbreak cases' contacts. The data demonstrate the potential of 0104 to be secondarily transmitted after long time periods - with some post-outbreak secondary cases occurring two to three months after disease onset (and clinical recovery) of the last known potential primary outbreak case. None of the confirmed post-outbreak cases developed HUS, but completeness of microbiological information on adult HUS cases remained problematic, even in the aftermath of the outbreak.

From the reported frequency of clearly outbreakunrelated infections with isolated $s x_{1}$-positive STEC (regardless of serotype) an overall increased testing volume for STEC, especially in the outbreak area, and especially in adults (Figure 2), can be gleamed. Testing frequency returned back to normal levels by the end of 2011 for adults, and even earlier in minors ( $\$ 18$ years of age). The capacity to detect infections with the outbreak strain is hampered by the incomplete serotyping of detected STEC (according to the German national database of mandatorily notifiable infections, $29 \%$ of STEC infections notified in 2012 in Germany were serotyped [9]). In 2012 and 2013 three further confirmed infections with STEC $\mathrm{O}_{104}$ were reported in Germany [9]. In the summer of 2012, an adult woman developed bloody diarrhoea two days after returning from Turkey - regrettably the ESBL-status of the infecting STEC 0104-strain was not determined. In 2013 two diarrhoeal STEC cases without travel history (a young boy and an adult woman) were notified as infected with different serotypes (0104: $\left.\mathrm{H}_{7}, \mathrm{O}_{104}: \mathrm{H}_{21}\right)$.

Importantly, the presented data do not support the notion that the outbreak strain has been able to establish itself in the German environment (which in turn could have been the source of further outbreaks). Just after the outbreak, this scenario had been a concern based on the observation that the outbreak strain can enter a viable but non-culturable state from which it can be resuscitated [13].

Regarding clinical picture, there was no post-outbreak case of HUS among the 17 confirmed symptomatic cases and only one among the 20 symptomatic postoutbreak cases that were either confirmed or probable. This contrasts with a stable proportion of $20 \%$ of the symptomatic outbreak cases developing this complication [2]. This finding may suggest a somewhat lower virulence of the outbreak strain in this period - possibly associated with the predominating non-alimentary uptake route or a weakening of the pathogen by passage. At the same time, many household transmissions, the post-outbreak cases among laboratory personnel and the nosocomial cluster demonstrate prolonged periods, during which secondary infections with the pathogen occurred. This is in line with long periods of shedding of STEC $0104: \mathrm{H}_{4}$ in outbreak cases, whereby shedding in an isolated case lasted longer than seven months [14]. 


\section{FIGURE 2}

Notified cases of Shiga toxin-producing Escherichia coli gastroenteritis without known serotype but positive for the $s t x_{1}$ gene, by reporting area and age group, Germany, January-December 2011

A. Minors, $₫ 18$ years-old

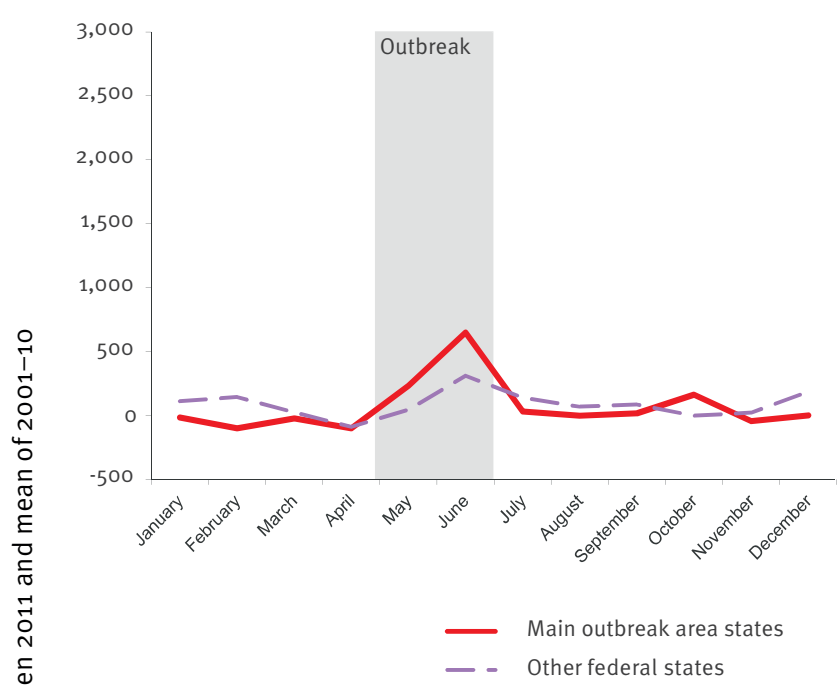

B. Adults, $\geq 18$ years-old

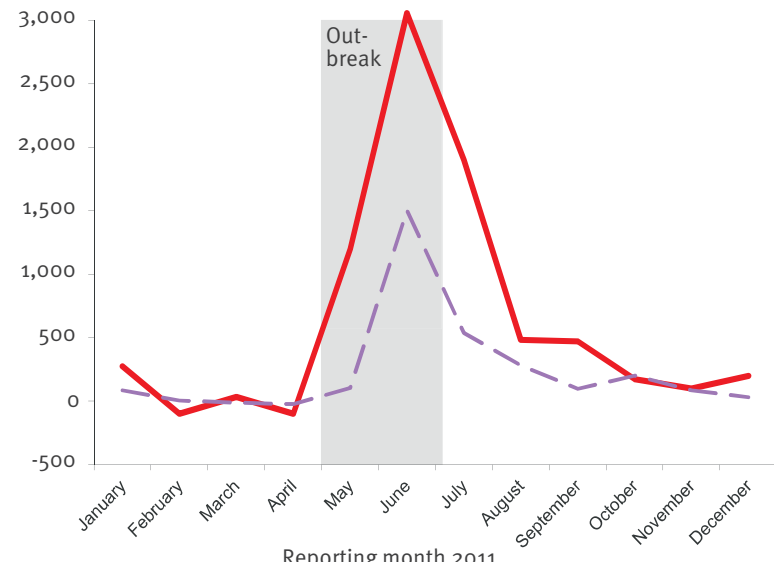

Sprout consumption was not recalled as a potential source of infection by any of the post-outbreak cases. Although fenugreek sprouts constitute a notoriously hard-to-remember food item, recall in the post-outbreak period would have been aided by the public announcement that these constituted the most likely source of infection in the outbreak. Also supporting the post-outbreak cases' stated non-consumption of sprouts is that for much of the post-outbreak period raw sprouts would hardly have been available in retail.

The two cases infected with non-outbreak related ESBLnegative STEC 0104: $\mathrm{H}_{4}$ strains in 2011 are intriguing, with both patients having likely acquired this infection in Turkey. In September 2011 in France infections with a similar strain were noted in a group of persons who had been in Turkey as tourists [10]. Together with the st $x_{1}$-only positive STEC $0_{104}: \mathrm{H}_{4}$ strain isolated from a child, these cases demonstrate existing variety among clinically relevant STEC 0104: $\mathrm{H}_{4}$.

A clinical surveillance of HUS has in the past proven very valuable in flagging STEC-associated disease outbreaks [15] and also gave first notice of this outbreak. For diarrhoeal illness, German STEC surveillance is mainly based on nonculture methods, which have the ability to quickly identify STEC [16]. However, strain information conveyed by most nonculture tests currently used in Germany is rudimental (Stx presence) and does not provide the relevant information to guide clinical management and epidemiological decisions.

\section{Acknowledgments}

The authors acknowledge the assistance of many colleagues in the outbreak team at RKI. We are grateful to many state and local health departments which collected the information on the cases presented here, as well as the laboratories and physicians who notified them, and the patients themselves who gave information to the health departments.

Conflict of interest

None declared.

Authors' contributions

Christina Frank coordinated post-outbreak surveillance, analysed the data and spearheaded writing of the manuscript. Astrid Milde-Busch was head of questionnaire data collection, in charge of data cleaning and participated in writing the manuscript. Dirk Werber conceptualised post-outbreak surveillance, provided microbiological background and participated in writing the manuscript.

\section{References}

1. Buchholz U, Bernard H, Werber D, Böhmer MM, Remschmidt C, Wilking $\mathrm{H}$, et al. German outbreak of Escherichia coli $\mathrm{O}_{104}: \mathrm{H}_{4}$ associated with sprouts. N Engl J Med. 2011;365(19):1763-70. http://dx.doi.org/10.1056/NEJMoa1106482

2. Frank C, Werber D, Cramer JP, Askar M, Faber M, an der Heiden $M$, et al. Epidemic profile of Shiga-toxin-producing Escherichia coli 0104:H4 outbreak in Germany. N Engl ] Med. 2011;365(19):1771-80. http://dx.doi.org/10.1056/ NEJMoa1106483

3. Robert Koch-Institut (RKI) Bericht: Abschließende Darstellung und Bewertung der epidemiologischen Erkenntnisse im EHEC 0104: $\mathrm{H}_{4}$ Ausbruch, Deutschland 2011. [Final presentation and evaluation of the epidemiological evidence in the EHEC 0104: H4 outbreak in Germany 2011]. Berlin: RKI; 2011. German.

4. Bielaszewska M, Mellmann A, Zhang W, Köck R, Fruth A, Bauwens A, et al. Characterisation of the Escherichia coli strain associated with an outbreak of haemolytic uraemic syndrome in Germany, 2011: a microbiological study. Lancet Infect Dis. 2011;11(9):671-6. http://dx.doi.org/10.1016/ S1473-3099(11)70165-7

5. Mellmann A, Harmsen D, Cummings CA, Zentz EB, Leopold SR, Rico A, et al. Prospective genomic characterization of the German enterohemorrhagic Escherichia coli 0104: $\mathrm{H}_{4}$ outbreak by rapid next generation sequencing technology. PLoS One. 2011;6(7):e22751. http://dx.doi.org/10.1371/journal. pone.0022751

6. Scheutz F, Nielsen EM, Frimodt-Moller J, Boisen N, Morabito $\mathrm{S}$, Tozzoli R, et al. Characteristics of the enteroaggregative Shiga toxin/verotoxin-producing Escherichia coli $\mathrm{O}_{104}: \mathrm{H}_{4}$ strain causing the outbreak of haemolytic uraemic syndrome 
in Germany, May to June 2011. Euro Surveill. 2011;16(24). pii: 19889.

7. Hauri A, Gotsch U, Strotmann I, Krahn J, Bettge-Weller G, Westbrock $\mathrm{H}$, et al. Secondary transmissions during the outbreak of Shiga toxin-producing Escherichia coli $0_{104}$ in Hesse, Germany, 2011. Euro Surveill. 2011;16(31). pii: 19937.

8. King LA, Nogareda F, Weill FX, Mariani-Kurkdjian P, Loukiadis E, Gault G, et al. Outbreak of Shiga toxin-producing Escherichia coli $\mathrm{O}_{104}: \mathrm{H}_{4}$ associated with organic fenugreek sprouts, France, June 2011. Clin Infect Dis. 2012;54(11):1588-94. http:// dx.doi.org/10.1093/cid/cis255

9. Robert Koch Institute (RKI). National data base of mandatorily notifiable infections, Germany, 2001 through 2013. Berlin: RKI.

10. Jourdan-da Silva N, Watrin M, Weill FX, King LA, Gouali M, Mailles A, et al. Outbreak of haemolytic uraemic syndrome due to Shiga toxin-producing Escherichia coli 0104: $\mathrm{H}_{4}$ among French tourists returning from Turkey, September 2011. Euro Surveill. 2012;17(4). pii: 20065.

11. Harendza S. "HUS diary" of a German nephrologist during the current EHEC outbreak in Europe. Kidney Int. 2011;80(7):687-9. http://dx.doi.org/10.1038/ki.2011.238

12. Loos S, Ahlenstiel T, Kranz B, Staude H, Pape L, Härtel C, et al. An outbreak of Shiga toxin-producing Escherichia coli $0_{104}: \mathrm{H}_{4}$ hemolytic uremic syndrome in Germany: presentation and short-term outcome in children. Clin Infect Dis. 2012;55(6):7539. http://dx.doi.org/10.1093/cid/cis531

13. Aurass P, Prager R, Flieger A. EHEC/EAEC $0104: \mathrm{H}_{4}$ strain linked with the 2011 German outbreak of haemolytic uremic syndrome enters into the viable but non-culturable state in response to various stresses and resuscitates upon stress relief. Environ Microbiol. 2011;13(12):3139-48. http://dx.doi. org/10.1111/j.1462-2920.2011.02604.x

14. Sin MA, Takla A, Flieger A, Prager R, Fruth A, Tietze E, et al. Carrier Prevalence, Secondary Household Transmission, and Long-Term Shedding in 2 Districts During the Escherichia coli 0104:H4 Outbreak in Germany, 2011. J Infect Dis.

2013;207(3):432-8. http://dx.doi.org/10.1093/infdis/jis702

15. Werber D, Frank C, Wadl M, Karch H, Fruth A, Stark K. Looking for tips to find icebergs--surveillance of haemolytic uraemic syndrome to detect outbreaks of Shiga toxin-producing E. coli infection. Euro Surveill. 2008;13(9):pii: 8053.

16. Jones TF, Gerner-Smidt P. Nonculture diagnostic tests for enteric diseases. Emerg Infect Dis. 2012;18(3):513-4. http:// dx.doi.org/10.3201/eid1803.111914 